\title{
Nitrogen Defects in Diamond Examined by an Electron Microprobe
}

\author{
N. C. Wilson ${ }^{1}$, C. M. MacRae ${ }^{1}$, A. Torpy ${ }^{1}$ and A. Tomkins ${ }^{2}$ \\ 1. CSIRO Mineral Resources, Microbeam Laboratory, Private Bag 10, Clayton South, Victoria, 3169 \\ 2. School of Earth Atmosphere and Environment, Monash University
}

Hyperspectral examination of diamonds in the electron probe microanalyser (EPMA) has proved a useful technique in the past to map nitrogen-vacancy defects and correlate these with elemental data[1]. In recent years, the data collection ability of the EPMA has greatly increased with the advent of new detectors, such as the soft x-ray emission spectrometer (SXES) [2] have not only lowered the limit of detectable X-rays to as low as Mg-L (49 eV), but also come with a high energy resolution, which can be as low as $99 \mathrm{meV}$ for $\mathrm{Mg} \mathrm{L}$. The importance of having high energy $\mathrm{x}$-ray resolution is that peak shape and position changes reflecting changes in the local chemical environment and can be easily observed for X-ray lines that are involved with bonding, such as the $\mathrm{C} \mathrm{K} \alpha$ line. SXES spectra are collected in parallel using a CCD camera, and in our case are collected simultaneously with WDS, EDS and CL spectral data.

CL spectra from diamonds are sensitive to defect structure, with more than 100 centres having been documented, giving rise to luminescence of more than a thousand spectral lines [3]. The resolution of the CL spectrometer is important for resolving defect lines, and the fixed grating spectrometers used for cathodoluminescence (CL) collection on EPMAs have also improved in specifications. These now offer the availability of higher resolution gratings and changeable entrance apertures, which leads to enhanced spectral resolution. The resolution of CL may be further improved by cooling the sample to liquid nitrogen $(\mathrm{LN})$ temperatures to reduce thermal broadening.

We have fitted a field-emission gun EPMA (JEOL 8530) with a LN stage, SXES detector, two SDD EDS detectors (Bruker XFLASH 6010) and a high resolution grating CL system [4]. The LN stage has the ability to move while at cryogenic temperature, so we can collect maps in both beam scan and stage scan. It is important to have a stage scanning capability, because in an EPMA the WD, CL and SXES spectrometers are optically aligned to a point and fully focusing. We have developed software to allow the simultaneous collection of the backscatter electron signal, WDS, EDS, SXES and CL spectral data. This simultaneous acquisition of all signals has the advantage of avoiding pixel misalignment and minimising electron beam induced damage artefacts associated with multiple pass mapping, in addition to that of the time saved.

The hyperspectral data sets collected can then be used to perform post-hoc examination to find unexpected phases (x-ray data) and defects or centres (CL data) within a sample. There are number of approaches that can be used to examine hyperspectral data sets, and in this work we have used clustering algorithms to partition and organise the hyperspectral data $[5,6]$.

Diamond from a range of sources have been examined, including carbonado diamonds and micro diamond inclusions in a meteorite sample (NWA3140). These samples have been mapped at both room and LN temperatures, with an example of a LN stage map from a carbonado diamond given in Fig 1a. The benefit of cooling to cryogenic temperatures in the LN cold stage can be seen in Fig. 1b which 
shows a CL spectrum from a carbonado diamond at room and LN temperatures. The structure of the phonon replicates for the nitrogen-vacancy defect at around 2.4 eV are sharper at LN temperatures [4].

References:

[1] N. C. Wilson et al, Microscopy and Microanalysis 18 (2012), p. 1303.

[2] M. Terauchi et al, 2012 Journal of Electron Microscopy 61(1), p. 1.

[3] Zaitsev, AM 2001, Optical Properties of Diamond, (Springer)

[4] We acknowledge support from ARC - LE130100087

[5] N. C. Wilson et al, Microscopy and Microanalysis 14(S2) (2008), p. 764.

[6] N. C. Wilson et al, Microscopy and Microanalysis 16(S2) (2010), p. 266.
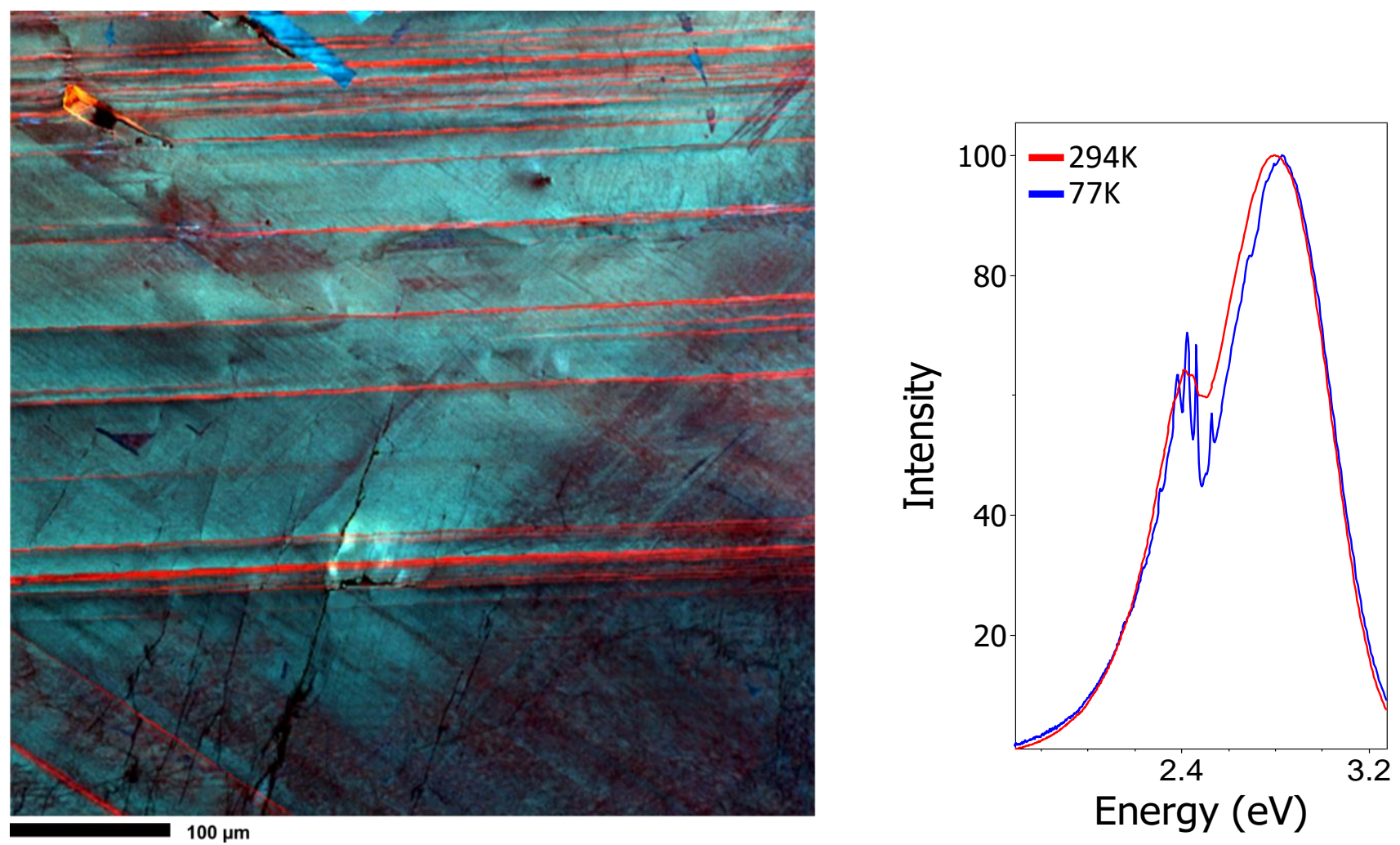

Figure 1. (a) Pseudo real-color CL map generated from a hyperspectral data set collected from a carbonado diamond at LN temperature using stage mapping. (b) Comparison of cathodoluminescence spectra from the same carbonado diamond collected at $294 \mathrm{~K}$ and $77 \mathrm{~K}$ (LN temperature). Spectra are normalised. 